\title{
Fronts propagating with signal dependent speed in Limited Diffusion and related Hamilton-Jacobi formulations
}

\author{
Susana Serna \\ Departament de Matematiques, Universitat Autonoma de Barcelona, 08193 Bellaterra, \\ Spain \\ Antonio Marquina \\ Departament de Matematica Aplicada, Universitat de Valencia, 46100 Burjassot, Spain
}

\begin{abstract}
We consider a class of limited diffusion equations and explore the formation of diffusion fronts as the result of a combination of diffusive and hyperbolic transport. We analyze a new class of Hamilton-Jacobi equations arising from the convective part of general Fokker-Planck equations ruled by a non-negative diffusion coefficient that depends on the unknown and on the gradient of the unknown. We explore the main features of the solution of the Hamilton-Jacobi equations that contain shocks and propose a suitable numerical scheme that approximates the solution in a consistent way with respect to the solution of the associated Fokker-Planck equation. We analyze three model problems covering different scenarios. One is the relativistic heat equation model where the speed of propagation of fronts is constant. A second one is a standard porous media model where the speed of propagation of fronts is a function of the density, is unbounded and can exceed any fixed value. We propose a third one which is a porous media model whose speed of propagating fronts depends on the density media and is limited. The three model problems satisfy a general Darcy law. We perform a set of numerical experiments under different piecewise smooth initial data with compact support and compare the behavior of the three different model problems.
\end{abstract}


Keywords: Limited diffusion equations, Hamilton-Jacobi equations, viscosity solutions with shocks, numerical approximation

\section{Introduction}

Many complex physical systems like plasmas, porous media, transport in statistical mechanics, geometric flows among others are described by a class of (anisotropic) diffusion equations that model transport by diffusion of a physical magnitude in a continuum medium $([1,2,3,4,7,17,18,19,22,23])$. Fokker-Planck formulation represents these models whose solutions contain diffusion fronts propagating with finite speed. Because of this feature these equations are known as limited diffusion equations.

Limited diffusion equations can be understood as an extension of Fourier theory of heat conduction ruled by the heat equation $u_{t}=\nu \Delta u$ where $\nu>0$ is the coefficient of heat diffusion in a specific media ([10]). The solution to this model for a delta initial signal is a Gaussian distribution defined over all $\mathcal{R}^{n}$ for any time $t$. The main drawback of this model relies on the fact that transport of heat conduction occurs at infinite speed making the model not appropriate for the description of many dissipative processes in thermodynamics. This issue has been widely discussed in the literature and different attempts to overcome it have been presented $([6,12,13,18,22])$.

We can understand the inadequacy of Fourier theory from the theory of relativity. Relativistic astrophysical flows are based on the assumption that light propagates with finite speed in vacuum, $c$, where $c$ is an upper bound of light speed in any other media. The principles of special relativity state that, to preserve causality, no physical signal can travel faster than $c([14])$. On the other hand, in non-relativistic physics it is advisable that the range of possible speeds of propagation be restricted to a validity region. In this work we explore mathematical models of transport prescribing a limited speed of propagation for the specific medium under study.

We consider the transport model represented by limited diffusion FokkerPlanck equations that written in conservation form read as

$$
u_{t}=\operatorname{div}(g(u,|\nabla u|) \nabla u)
$$

where $g(u, p)$ is a non-negative real function of $u \geq 0$ and $|\nabla u|$. This transport model allows the formation of diffusion fronts propagating with finite speed under certain conditions on function $g$ and the solution for a compact 
supported initial data is confined to a bounded region in $\mathcal{R}^{n}$ at any time $t>0([1,2,4,5,18])$.

In our approach we analyze a particular class of limited diffusion FokkerPlanck equations of type (1). We study the dynamics of the solution of the model in terms of their convective and diffusive terms and investigate the speed of propagation of diffusion fronts for three model examples.

The simplest model of transport with finite speed of propagation is represented by the hyperbolic model where finite speeds are intrinsically prescribed from real eigenvalues of the Jacobian of the fluxes. Hyperbolic transport prescribes a finite speed of propagation of fronts, ([15]).

For the purpose of analyzing the transport in limited diffusion FokkerPlanck equations we expand spatial derivatives of the flux equation (1) obtaining the expression

$$
u_{t}=\frac{\partial g}{\partial u}|\nabla u|^{2}+g(u,|\nabla u|) \Delta u+\frac{\partial g}{\partial p} \frac{L(u)}{|\nabla u|}
$$

where $p$ represents the second argument of function $g$ and

$$
L(u)=u_{x}^{2} u_{x x}+2 u_{x} u_{y} u_{x y}+u_{y}^{2} u_{y y}
$$

is a second order nonlinear elliptic operator expressing the second derivative of $u$ in the direction of the gradient.

Expression (2) reveals that propagation of fronts in limited diffusion equations of the form (1) is the result of a combination of nonlinear effects that include hyperbolic convection (first term) and transport by anisotropic diffusion (second and third terms). Therefore we can state that convective dynamics and possible formation of shocks are ruled according to a hyperbolic law, the first term in (2), which in this case is a Hamiltonian.

In this work we study the Hamiltonian term as the convective part of the Fokker-Planck equations and the cause of the formation of shocks in the solution of the limited diffusion equations. In our study we consider a new class of Hamilton-Jacobi equations associated to the Hamiltonian in (2) as

$$
u_{t}=\frac{\partial g}{\partial u}|\nabla u|^{2}
$$

that result from removing diffusion terms in (2). We investigate the formation of shocks in the solution of these Hamilton-Jacobi equations. 
We study three model problems of limited diffusion equations namely: the relativistic heat equation, a standard porous media equation and a limited speed porous media equation. The model problems satisfy a general Darcy law and cover three different scenarios: one prescribing a constant speed of propagation of diffusion fronts; a second case where diffusion fronts propagate with unbounded finite speed and a third case where diffusion fronts propagate with density dependent speed bounded in magnitude by an established reference speed $C$.

We analyze the different behaviors of the limited diffusion equations throughout their Hamilton-Jacobi counterparts. We perform a set of numerical experiments and compare the solution for the three model problems for the limited diffusion equations and their corresponding Hamilton-Jacobi equations. For the numerical approximation of the solution of the limited diffusion Fokker-Planck equations we use the conservative numerical scheme presented in [18]. For the numerical approximation of the new class of Hamilton-Jacobi equations we propose a suitable finite difference numerical scheme to approximate the viscosity solution with shocks arising in the models. The new numerical scheme is designed as an extension of the standard classical schemes that only approximate viscosity solutions of HamiltonJacobi equations that develop discontinuities in first derivative [9, 20, 21, 27]. The proposed numerical scheme combines the properties of classical numerical schemes for Hamilton-Jacobi equations together with the features of a shock capturing scheme to ensure formation of shocks and correct propagation of waves.

The paper is organized as follows. In section 2 we present three model problems of a class of limited diffusion equations and analyze the behavior of their convective terms as Hamilton-Jacobi equations that develop shocks in the solution. Section 3 is devoted to presenting a suitable numerical scheme for the approximation of the viscosity solution with shocks associated to the Hamilton-Jacobi equations. In section 4 we perform numerical experiments for different initial data. Section 5 includes our conclusions. 


\section{Hamilton-Jacobi equations arising from limited diffusion equa- tions}

We focus on limited diffusion Fokker-Planck equations of type (1) where the function $g$ is defined as

$$
g(u,|\nabla u|)=f(u) \frac{r}{\sqrt{u^{2}+r^{2}|\nabla u|^{2}}}, \quad r=\frac{\nu}{C},
$$

with flux $f(u)$ convex and a positive function of $u, C>0$ an established reference speed and $\nu>0$ the kinematic viscosity in a specific medium. The limited diffusion equation (1) reads then as

$$
u_{t}=\operatorname{div}\left(\frac{f(u) r \nabla u}{\sqrt{u^{2}+r^{2}|\nabla u|^{2}}}\right)
$$

and its convective Hamiltonian term as

$$
\frac{r|\nabla u|^{2}}{\sqrt{u^{2}+r^{2}|\nabla u|^{2}}}\left(f^{\prime}(u)-\frac{u f(u)}{u^{2}+r^{2}|\nabla u|^{2}}\right)
$$

From (7) and following (4) we define a new class of Hamilton-Jacobi equations arising from limited diffusion equations of type (6) as

$$
u_{t}=\frac{r|\nabla u|^{2}}{\sqrt{u^{2}+r^{2}|\nabla u|^{2}}}\left(f^{\prime}(u)-\frac{u f(u)}{u^{2}+r^{2}|\nabla u|^{2}}\right)
$$

Let us perform a heuristic asymptotic analysis of the limited diffusion equation (6) in one spatial dimension.

In one dimension the limited diffusion Fokker-Planck equation with the flux $f(u)$ can be expressed as

$$
u_{t}=\left(\frac{f(u) r u_{x}}{\sqrt{u^{2}+r^{2} u_{x}^{2}}}\right)_{x}
$$

where the non-divergence form of this equation is

$$
u_{t}=\frac{r u_{x}^{2}}{\sqrt{u^{2}+r^{2} u_{x}^{2}}}\left(f^{\prime}(u)-\frac{u f(u)}{u^{2}+r^{2} u_{x}^{2}}\right)+f(u) \frac{r u^{2}}{\left(u^{2}+r^{2} u_{x}^{2}\right)^{3 / 2}} u_{x x}(10)
$$


The convective part written as a Hamilton-Jacobi equation is

$$
u_{t}=\frac{r u_{x}^{2}}{\sqrt{u^{2}+r^{2} u_{x}^{2}}}\left(f^{\prime}(u)-\frac{u f(u)}{u^{2}+r^{2} u_{x}^{2}}\right)
$$

We wish to identify the dynamics of propagating fronts in both limited diffusion and Hamilton-Jacobi equations, (9) and (11) respectively. Let us assume that around jump discontinuities or "large gradients" where $\left|u_{x}\right|>>$ $u$, the ratio $\frac{u}{r\left|u_{x}\right|}<<1$ is small. Then for $y=\left(\frac{u}{r u_{x}}\right)^{2}$ and using Taylor expansion of $(1+y)^{-\frac{1}{2}}=1-\frac{1}{2} y+\frac{3}{4} y^{2}-\cdots($ convergent for $|y|<1)$ we have that

$$
\left(1+\left(\frac{u}{r u_{x}}\right)^{2}\right)^{-1 / 2}=1-\frac{1}{2}\left(\frac{u}{r u_{x}}\right)^{2}+\frac{3}{4}\left(\frac{u}{r u_{x}}\right)^{4}-\cdots
$$

Hence, defining $\operatorname{sgn}\left(u_{x}\right)=\frac{u_{x}}{\left|u_{x}\right|}$, around jumps we obtain

$$
u_{t}=\left(f(u) \frac{r u_{x}}{\sqrt{u^{2}+r^{2} u_{x}^{2}}}\right)_{x}=\left(f(u) \operatorname{sgn}\left(u_{x}\right)\left(1+\left(\frac{u}{r u_{x}}\right)^{2}\right)^{-1 / 2}\right)_{x}
$$

that resembles a hyperbolic conservation law like

$$
u_{t} \approx\left(f(u) \operatorname{sgn}\left(u_{x}\right)\right)_{x}
$$

ruling the dynamics of the diffusion fronts in (9). This means that shock waves may be generated in finite time at the boundaries of the support of the solution.

On the other hand, and disregarding the term

$$
\frac{u f(u)}{u^{2}+r^{2} u_{x}^{2}} \approx O\left(\left(\frac{u}{r u_{x}}\right)^{2}\right)
$$

equation (11) around jump discontinuities resembles

$$
u_{t} \approx \operatorname{sgn}\left(u_{x}\right) f^{\prime}(u) u_{x}
$$

because it follows that, if $\left|\frac{u}{r u_{x}}\right|<<1$, then

$$
\frac{r u_{x}}{\sqrt{u^{2}+r^{2} u_{x}^{2}}} \approx \operatorname{sgn}\left(u_{x}\right)
$$


From this analysis we conclude that propagation fronts in limited diffusion equations of type (9) behave according to the features of a hyperbolic conservation law with hyperbolic flux $f(u)$ and their speed of propagation is determined approximately from $\operatorname{sgn}\left(u_{x}\right) f^{\prime}(u)$.

In what follows we analyze three different types of model problems of limited diffusion equations corresponding to three possible definitions of the flux $f(u)$. We consider the one dimensional limited diffusion equation (9) and the Hamilton-Jacobi counterparts for the hyperbolic fluxes $f(u)=C u$, $f(u)=C \frac{u^{2}}{2}$ and $f(u)=C(u-\log (1+u))$. The first flux is a linear flux and the other two are nonlinear and convex. Therefore the only possible jump discontinuities for the latter two cases are shock waves ([14]).

\subsection{Relativistic Heat Equation}

With the hyperbolic flux $f(u)=C u$, with $C=c$ representing an established reference speed, the limited diffusion Fokker-Planck equation (6) becomes the so-called relativistic heat equation,

$$
u_{t}=\nu \operatorname{div}\left(\frac{u \nabla u}{\sqrt{u^{2}+\left(\frac{\nu}{c}\right)^{2}|\nabla u|^{2}}}\right)
$$

where $\nu>0$ and $r=\frac{\nu}{c}$.

This equation represents a mathematical model describing transport by diffusion with finite speed of propagation. It was proposed by Rosenau to overcome limitations of Fourier heat conduction classical theory ([22]). Fourier model written in conservation form is expressed as

$$
u_{t}=-\nabla(u \vec{v})
$$

where the velocity field $\vec{v}=-\nu \frac{\nabla u}{u}$ is proportional to the possibly unbounded gradient of $u$. Rosenau proposed to change the velocity field in (15) by

$$
\vec{v}_{R}=-\frac{\nu \nabla u}{\sqrt{u^{2}+\left(\frac{\nu}{c}|\nabla u|\right)^{2}}}
$$

so it is bounded by $c([22])$. Then equation (15) becomes the relativistic heat equation (14).

The relativistic heat equation has been widely studied from the analytical point of view $[1,2,3,4,18,22]$ and more recently a numerical approach has been presented in [18]. In the later the author proposes a conservative 
numerical scheme for the approximation of a specific class of Fokker-Planck equations in which the relativistic heat equation is a model problem.

In one dimension, the relativistic heat equation reads as

$$
u_{t}=\nu\left(\frac{u u_{x}}{\sqrt{u^{2}+r^{2} u_{x}^{2}}}\right)_{x}, \quad t>0, u \geq 0
$$

and its convective part, the Hamilton-Jacobi equation, is

$$
u_{t}=c\left(\frac{r u_{x}}{\sqrt{u^{2}+r^{2} u_{x}^{2}}}\right)^{3} u_{x}
$$

From the asymptotic analysis performed previously in this section we can assert that equation (17) behaves as a hyperbolic conservation law with flux $\operatorname{sgn}\left(u_{x}\right) c u$. Therefore, diffusion fronts in (17) and shock waves in (18) propagate at finite speed with constant value $c>0$ according to the direction prescribed by the sign of $u_{x}$. An analytical proof of this result can be found in $[1]$.

The relativistic heat equation overcomes limitations of heat conduction classical model by Fourier by prescribing a finite speed of propagation. However the description is still not realistic since it is not able to represent diffusion phenomena where the speed of propagation depends on the density of the moving quantity.

Let us consider a hyperbolic flux $f(u)$ that is nonlinear with respect to $u$.

\subsection{Porous-media equation}

Setting the hyperbolic flux $f(u)=C \frac{u^{m}}{m}$ with $m>1$ and $C$ an established reference speed, the limited diffusion equation (6) becomes the socalled porous-media equation $([1,2])$. The convective hyperbolic transport of the porous media equation was studied in [18] and more recently in [5] where it is proved that diffusion fronts propagate according to Rankine-Hugoniot relations. In one dimension the porous media equation reads as

$$
u_{t}=\operatorname{div}\left(\frac{\nu u^{m} u_{x}}{m \sqrt{u^{2}+r^{2}\left|u_{x}\right|^{2}}}\right) \quad \nu>0, m>1
$$

and the corresponding Hamilton-Jacobi porous-media equation as

$$
u_{t}=C u^{m-1} \frac{r\left|u_{x}\right|^{2}}{\sqrt{u^{m}+r^{2}\left|u_{x}\right|^{2}}}\left[m-\frac{u^{2}}{u^{2}+r^{2}\left|u_{x}\right|^{2}}\right]
$$


Following the previously presented asymptotic analysis, limited diffusion porous media equation (19) behaves around jump discontinuities as

$$
u_{t} \approx\left(C \frac{u^{m}}{m} \operatorname{sgn}\left(u_{x}\right)\right)_{x}
$$

and the Hamilton-Jacobi one as

$$
u_{t} \approx C u^{m-1} \operatorname{sgn}\left(u_{x}\right)
$$

Thus, when present, diffusion fronts propagate with speed proportional to $u^{m-1}$. In this case the speed of propagation is finite although an unbounded function of the density of the moving quantity. The moving quantity can travel with a speed exceeding any fixed value, including $C$. In our experiments we will consider the case for $m=2$.

Next we propose a nonlinear hyperbolic flux $f(u)$ generating a limited diffusion equation where diffusion fronts propagate with bounded non constant speed.

\subsection{Limited speed porous media equation}

Considering the hyperbolic flux $f(u)=C(u-\log (1+u))$ in (6) where $C$ represents an established reference speed, the limited diffusion Fokker-Planck equation becomes what we call the limited speed porous media equation. In one dimension it is expressed as

$$
u_{t}=\left(\frac{C(u-\log (1+u)) r u_{x}}{\sqrt{u^{2}+r^{2} u_{x}^{2}}}\right)_{x}
$$

The convective term written as a Hamilton-Jacobi equation reads as

$$
u_{t}=\frac{r u_{x}^{2}}{\sqrt{u^{2}+r^{2} u_{x}^{2}}}\left(\frac{C u}{1+u}-\frac{C u(u-\log (1+u))}{u^{2}+r^{2} u_{x}^{2}}\right)
$$

From the asymptotic analysis performed at the beginning of the section we observe that diffusion fronts in (23) and shock waves in (24) propagate with speed proportional to

$$
f^{\prime}(u)=\frac{C u}{1+u}
$$


which is bounded by $C$. Therefore the speed of propagation of fronts depends on the moving quantity and is bounded in magnitude by an established reference speed $C>0$.

Remark: An observation on the differences of the hyperbolic fluxes presented in this section is the following. Possible jump discontinuities for the case where $f(u)$ is linear (relativistic heat equation case) are linear discontinuities where characteristic curves are parallel. The cases where the hyperbolic flux $f(u)$ is nonlinear with respect to $u$ (porous media cases) the characteristic curves intersect and therefore jump discontinuities are shock waves.

The three model examples of Fokker-Planck equations analyzed in this section are particular cases of a general Darcy law. Following Rosenau ([22]) we can formulate a general Darcy law expressed as the relation

$$
\nabla P=\frac{-\mu(\rho) \vec{v}}{\sqrt{1-\frac{v^{2}}{V^{2}}}}
$$

where $P$ represents the pressure (which is a function of the density $\rho$ ), $\vec{v}$ the velocity field, $|\vec{v}|^{2}=v^{2}$ and $\mu(\rho)$ is the permeability coefficient defined as

$$
\mu(\rho)=\frac{\rho}{\nu}
$$

with $\nu>0$ the kinematic viscosity. $V=V(\rho)$ is an upper bound of the speed of the medium defined as

$$
V=C P^{\prime}(\rho)
$$

where $C>0$ is a reference speed of the porous medium and $P^{\prime}(\rho)>0$ the derivative of the pressure with respect to $\rho$.

From (26) we can express the continuity equation as

$$
\rho_{t}=\operatorname{div}(-\rho \vec{v})=\operatorname{div}\left(\frac{\rho \nabla P}{\sqrt{\mu(\rho)^{2}+\frac{|\nabla P|^{2}}{V^{2}}}}\right)
$$

Since $\nabla P=P^{\prime}(\rho) \nabla \rho$ we can retrieve the Fokker-Planck equation as

$$
\rho_{t}=\operatorname{div}\left(\frac{C \rho P^{\prime}(\rho) r \nabla \rho}{\sqrt{\rho^{2}+r^{2}|\nabla \rho|^{2}}}\right)
$$

where $r=\frac{C}{\nu}>0$. 
As particular cases of the general Darcy law (26) we recover the three model examples studied previously in this section. Indeed, for $P^{\prime}(\rho) \equiv 1$ $(P(\rho)=\rho)$ we obtain the relativistic heat equation and if $P^{\prime}(\rho) \equiv \frac{\rho}{2}$ we get the standard porous media equation. The limited speed porous media equation is obtained by setting $P^{\prime}(\rho) \equiv 1-\frac{\log (1+\rho)}{\rho}$. In the later case $V(\rho) \leq$ $C$ is satisfied for every $\rho>0$.

In order to observe the behavior of the dynamics of the presented model problems our next goal consists of performing numerical simulations of the evolution of their solution. We will compare the results obtained for the limited diffusion equations with the ones for the Hamilton-Jacobi counterparts for each model problem and for different initial data.

\section{Numerical approximation of the Fokker-Planck and Hamilton- Jacobi models}

In this section we describe the numerical schemes we use for the approximation of the solution of the limited diffusion equations and their HamiltonJacobi counterparts studied in previous section. The general set up is as follows.

We consider one dimensional domains, $x \in[a, b]$ and initial value $u(x, 0)=$ $u_{0}(x)$ and homogeneous Neumann boundary conditions. We settle the computational grid as $x_{j}=a+j h, j=0, \cdots, N, h=\frac{b-a}{N}$ where $N$ is the number of subintervals of the partition. We extend the index domain from $j=-1$ to $N+1$ where $x_{-1}=a-h$ and $x_{N+1}=b+h$. We denote by $\Delta t$ the time step size and $t_{n}:=n \Delta t$. By $u_{j}^{n}$ we represent the numerical approximation of the solution $u\left(x_{j}, t_{n}\right)$ with $u_{j}^{0}=u_{0}\left(x_{j}\right), j=0,1, \cdots, N$. Homogeneous Neumann boundary conditions are enforced at every time step $t_{n}$ by setting $u_{-1}^{n}=u_{0}^{n}$ and $u_{N+1}^{n}=u_{N}^{n}$.

\subsection{Conservative numerical scheme for limited diffusion models}

For the numerical approximation of the solution of the limited diffusion Fokker-Planck equations considered in the previous section we use the conservative finite difference numerical scheme proposed in [18]. The explicit numerical scheme applies to limited diffusion equations written in divergence form as

$$
u_{t}=\left(g\left(u,\left|u_{x}\right|\right) u_{x}\right)_{x}
$$


with $g$ as described in (5), ([18]). The numerical scheme reads as

$$
\begin{aligned}
u_{j}^{n+1}= & u_{j}^{n}+\frac{\Delta t}{h^{2}}\left(g\left(\frac{u_{j}^{n}+u_{j+1}^{n}}{2},\left|\frac{u_{j+1}^{n}+u_{j}^{n}}{h}\right|\right)\left(u_{j+1}^{n}+u_{j}^{n}\right)\right. \\
& \left.-g\left(\frac{u_{j-1}^{n}+u_{j}^{n}}{2},\left|\frac{u_{j}^{n}+u_{j-1}^{n}}{h}\right|\right)\left(u_{j}^{n}+u_{j-1}^{n}\right)\right)
\end{aligned}
$$

As demonstrated in [18] the explicit numerical scheme is consistent, stable, conserves mass exactly, preserves positivity and satisfies a discrete local maximum principle. The Courant-Friedrichs-Lewy (CFL) ([8]) time step restriction to ensure stability is a parabolic condition of the form

$$
\frac{\Delta t}{h^{2}} \leq \frac{1}{2 K}
$$

where $K=\max |g(u, p)|$ in the domain of the solution ([18]). We assume $g(u, p)$ is bounded for all $u$ and $p$ in the domain.

\subsection{Numerical scheme for Hamilton-Jacobi equations developing shocks}

Classical theory by Crandall and Lions ([9]) on viscosity solutions of Hamilton-Jacobi equations ensures existence of a unique global continuous solution in time (with possible discontinuities in derivative). Standard numerical schemes for the approximation of classical viscosity solutions of Hamilton-Jacobi equations are designed to develop continuous approximations $([20,21,27])$ and therefore are not appropriate for the approximation of solutions with shocks.

In this section we introduce a new finite difference numerical scheme to approximate the solution of the new class of Hamilton-Jacobi equations (11) where shocks are allowed in the solution. The numerical scheme is based on a new class of shock capturing numerical Hamiltonians.

In order to motivate the design of the new numerical scheme we first review the main facts on the classical theory on continuous viscosity solutions and their numerical approximation.

Classical theory on viscosity solutions ensures existence of a unique global continuous solution in time for the initial value problem

$$
u_{t}=H(u, \nabla u), \quad u(x, 0)=u_{0}(x)
$$

for continuous initial data provided the Hamiltonian $H$ is continuous and

$$
u \rightarrow H(u, p)
$$


is a non increasing function for each $p \in \mathcal{R}^{n}$.

Standard numerical schemes in one dimension for the numerical approximation of viscosity solutions of (34) are designed from Lipschitz, monotone and consistent numerical Hamiltonians $\tilde{h}$. These depend on three variables, $\tilde{h}\left(u, p^{-}, p^{+}\right)$, where $p^{-}=\frac{u_{j}^{n}-u_{j-1}^{n}}{h}$ and $p^{+}=\frac{u_{j+1}^{n}-u_{j}^{n}}{h}$. A first order numerical scheme in one dimension for approximating the solution of (34) reads as

$$
u_{j}^{n+1}=u_{j}^{n}+\Delta t \tilde{h}\left(u_{j}^{n}, p^{-}, p^{+}\right),
$$

The scheme is stable under a CFL condition

$$
\frac{\Delta t}{h} \leq k_{0} \frac{1}{\max _{u, p}\left|H(u, p)_{p}\right|}
$$

where $k_{0}<1$ and converges to the classical viscosity solution provided $\tilde{h}$ is monotone and consistent, i.e., $\tilde{h}(u, p, p)=H(u, p)$, ([27]).

In our study we are considering Hamilton-Jacobi equations of the form

$$
u_{t}=G(u,|\nabla u|)|\nabla u|^{2}
$$

where $G(u, p)=\frac{\partial g}{\partial u} \geq 0, g$ defined as in (5), i.e., $g(u, p)=f(u) S(u, p)$, $f(u)>0$, convex and $S(u, p)=\frac{r}{\sqrt{u^{2}+r^{2} p^{2}}}$ with $r>0$.

The Hamiltonian in $(38), H(u, p)=G(u, p) p^{2}$, does not satisfy monotonicity condition for every $u$ since the partial derivative $\frac{\partial H}{\partial u}$ may change sign as a function of $u$ for constant $p$. It follows from expression

$$
\frac{\partial^{2} g}{\partial u^{2}}=f^{\prime \prime}(u) S(u)-\frac{S(u)^{3}}{r^{2}}\left(2 u f^{\prime}(u)+f(u)\right)+\frac{3 u^{2}}{r^{4}} S(u)^{5} f(u)
$$

The sign of $\frac{\partial^{2} g}{\partial u^{2}}$ depends on the choice of $f(u)$ since $S(u)>0$ and $f^{\prime \prime}(u)>0$ ( $f(u)$ convex).

In the cases where $f(u)$ induces non monotonicity to the Hamiltonian in (38) the solution may develop jump discontinuities in finite time. This can be proved following the method of characteristics ([15]). Hence conventional theory of viscosity solutions by Crandall and Lions does not apply to Hamilton-Jacobi equations of type (38). On the other hand convergence of the numerical scheme (36) is not ensured in the presence of jump discontinuities. The numerical scheme fails to be monotone when the monotonicity condition on the Hamiltonian in (38) with respect to $u$ is not satisfied. 
The three Hamiltonians under study defined from functions $f(u)$ for the cases described in subsections 2.1, 2.2 and 2.3 are non monotone with respect to $u$. We seek to simulate through numerical approximation the formation of shocks in the solution of the Hamilton-Jacobi equations deduced from limited diffusion models.

In the following we design a numerical scheme based on a shock capturing numerical Hamiltonian that combines the properties of classical numerical schemes for Hamilton-Jacobi equations as $(36)$, ([20, 26, 27]), together with the features of a shock capturing scheme to ensure formation and correct propagation of waves $([16,26])$. In particular we design a shock capturing numerical Hamiltonian by using a Godunov type strategy where the upwind component is plugged into the unknown $u$ and gradients are approximated by means of a local monotone scheme. We propose numerical Hamiltonians with four arguments taking into account the flow of information contained in the unknown $u$. Indeed, the numerical Hamiltonian will depend at each location $x_{j}$ on the arguments $u^{-}, u^{+}, p^{-}, p^{+}$where $u^{-}=\frac{u_{j-1}^{n}+u_{j}^{n}}{2}, u^{+}=\frac{u_{j}^{n}+u_{j+1}^{n}}{2}, p^{-}=$ $\frac{u_{j}^{n}-u_{j-1}^{n}}{h}$ and $p^{+}=\frac{u_{j+1}^{n}-u_{j}^{n}}{h}$.

Thus, the numerical scheme for the approximation of viscosity solutions with shocks is defined as

$$
u_{j}^{n+1}=u_{j}^{n}+\Delta t \tilde{h}\left(u^{-}, u^{+}, p^{-}, p^{+}\right)
$$

where the numerical Hamiltonian $\tilde{h}\left(u^{-}, u^{+}, p^{-}, p^{+}\right)$is consistent with (38) i.e.,

$$
\tilde{h}(u, u, p, p)=\frac{\partial g}{\partial u}(u, p) p^{2}
$$

The first order accurate shock capturing numerical Hamiltonian is defined as follows. We apply the Osher-Sethian numerical Hamiltonian strategy ([20]) when gradients of $u_{j}$ in both directions (represented by $p^{-}, p^{+}$) share sign. For the cases where $p^{-}$and $p^{+}$have different signs we apply Godunov shock capturing strategy $([11,26])$, i.e., zero flux when the characteristic curves do not intersect (rarefaction waves) and upwind option otherwise depending on the sign of the local speed defined as a function of $p^{-}$and $p^{+}$.

The algorithm, considering $u, v, p$ and $q$ real numbers, reads as:

if $(p \geq 0)$ and $(q \geq 0)$

$$
\tilde{h}(u, v, p, q)=G(v, q) q^{2}
$$

else

$$
\text { if }(p<0) \text { and }(q<0)
$$


else

$$
\tilde{h}(u, v, p, q)=G(u, p) p^{2}
$$

$$
\text { if } \begin{gathered}
(p<0) \text { and }(q \geq 0) \\
\tilde{h}(u, v, p, q)=0
\end{gathered}
$$

else

$$
\begin{aligned}
& s=\frac{|q|-|p|}{q-p} \\
& \text { if } s>0 \\
& \quad \tilde{h}(u, v, p, q)=G(v, q) q^{2}
\end{aligned}
$$

else

$$
\tilde{h}(u, v, p, q)=G(u, p) p^{2}
$$

end

end

end

end

The new numerical scheme allows formation of shocks and correct propagation of waves. The proposed first order numerical scheme for HamiltonJacobi equations developing shocks is stable under a hyperbolic CFL time step size restriction that combines the CFL restrictions for shock capturing and classical Hamilton-Jacobi numerical schemes. It reads as the minimum of both as

$$
\frac{\Delta t}{h} \leq k_{0} \min \left(\frac{1}{\max _{u, p}\left|H(u, p)_{p}\right|}, \frac{1}{\max _{u}\left|f^{\prime}(u)\right|}\right)
$$

where $k_{0}<1$. Let us note that in all our cases $(H(u, p))_{p}$ is uniformly bounded for each fixed $u$, and $p$ in $[0, \infty[$.

The following section is devoted to perform numerical approximations of the presented limited diffusion equations and their Hamilton-Jacobi counterparts.

\section{Numerical experiments}

In this section we show numerical evidence of the analysis performed in section 2 presenting a set of numerical experiments using the numerical schemes introduced in the previous section. We compute the solution and examine the evolution of each of the model problems for the relativistic heat, porous media and limited speed porous media Fokker-Planck equations and the associated Hamilton-Jacobi equations for a set of one-dimensional initial data. We also compare the solutions of the models in terms of their behavior 
with respect to the speed of propagation of diffusion fronts under different initial data.

We present three examples with initial data defined in $x \in[-3,3]$ and consider a partition of 500 grid points for each numerical approximation. We set constant $C=1$ for the three model problems. We run all experiments at fifth order of accuracy. For the extension to fifth order accuracy in space in the approximation of the solution of the limited diffusion Fokker-Planck equations we proceed as explained in [18] using the Weighted PowerENO reconstruction procedure ([24]).

Fifth order of accuracy in space for the Hamilton-Jacobi cases is obtained from the basic first order scheme by computing high-order approximations to arithmetic means $u^{-}$and $u^{+}$and to the gradients at both sides of the node $x_{j}, p^{-}$and $p^{+}$by means of a reconstruction procedure $([21])$. We use the Weighted PowerENO fifth order reconstruction procedure. The implementation for Hamilton-Jacobi equations is detailed in [25].

High order accuracy in time is obtained in both cases by using an explicit third order TVD Runge-Kutta scheme ([26]).

In order to ensure stability of the numerical scheme for the three limited diffusion models we set, according to (33), an upper bound of the CFL restriction fixing $\Delta t=0.25 * h^{2}$. Stability of the numerical scheme for the approximation of the solution of the Hamilton-Jacobi cases is ensured by setting and upper bound to the CFL restriction according to (37) taking $\Delta t=0.1 * h$.

\subsection{Example 1: Double step initial data}

We consider an initial data consisting of a piecewise constant function containing jump discontinuities at the boundaries of the support and two jump discontinuities at the interior of the support. The initial data is defined as

$$
u_{0}(x)= \begin{cases}2 & |x| \leq 1 \\ 1 & -2 \leq x<-1 \\ 1 & 1 \leq x \leq 2 \\ 0 & \text { elsewhere }\end{cases}
$$

Numerical results for times $0.01,0.3$ and 0.75 are displayed in Figure 1. Left column includes the approximate solutions to the limited diffusion FokkerPlanck versions of each model problem and right column includes their Hamilton-Jacobi counterparts. From top to bottom the models are: relativistic heat, porous media and limited speed porous media equations. 

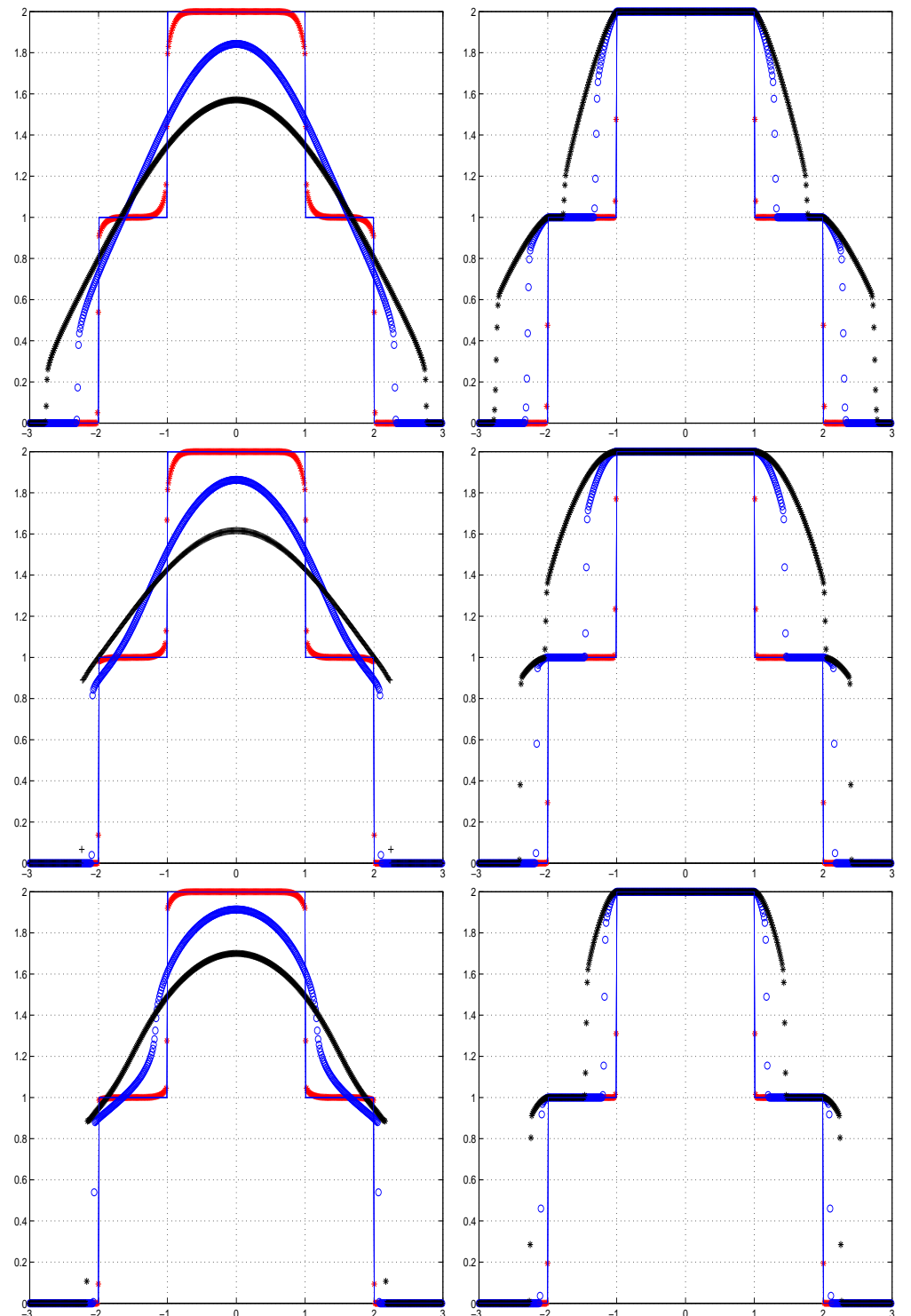

Figure 1: Double step initial data. Evolution at time, $t=0.01,0.3$ and $t=0.75$ represented by red "*" blue "o" and black "+" respectively. Top to bottom, relativistic heat, porous media and limited speed porous media equations. Left column, limited diffusion equations, right column, Hamilton-Jacobi counterparts. 
Left column of Figure 1 displays the three cases of limited diffusion Fokker-Planck equations. Transport by diffusion occurs in the interior of the support which expands with finite speed. We observe how diffusion smears interior jump discontinuities from early times of the evolution. Diffusive effect is stronger in the relativistic heat equation case (first row) where at larger times diffusion completely dissolves inner discontinuities.

Hyperbolic transport is localized at the boundaries of the support. Magnitude of diffusion fronts vary along the evolution depending on the diffusion rate. The speed of diffusion fronts at the boundaries of the support in the relativistic heat equation case (first row) is constant equal to 1 . In the second row the speed of diffusion fronts for porous media limited diffusion equation is the one prescribed by the Rankine-Hugoniot relations for the hyperbolic flux (as mentioned in subsection 2.2). In the last row, for the case of the limited speed porous media equation, the speed of diffusion fronts depends on $u$ and behaves similarly to the porous media case.

We observe differences on the numerical resolution of jump discontinuities at the boundary of the support for the relativistic heat equation case (first row) and porous media cases (second and third rows). These differences are justified since, as remarked in section 2, jump discontinuities in the relativistic heat equation case are linear discontinuities which are advected with constant speed while jump discontinuities in the porous media cases are shock waves that propagate with speed magnitude proportional to the derivative of the corresponding flux.

Right column of Figure 1 displays the results obtained for the HamiltonJacobi associated equations. We observe a completely different dynamics in the evolution compared to the one for the limited diffusion equation. In the absence of diffusive effects, the three cases present shocks, linear discontinuities and rarefactions waves as expected in a hyperbolic flux ([15]). We observe in the first row that the support of the initial data for the relativistic heat equation expands with same speed as the one for the corresponding limited diffusion equation (left column). For the porous media Hamilton-Jacobi equations diffusion fronts propagate faster than in the corresponding limited diffusion cases since the magnitude of the shocks are bigger due to the lack of dissipation.

\subsection{Example 2: Semi-circle wave problem}

We consider an initial data with compact support consisting on a semicircle with radius equal to 2 , 

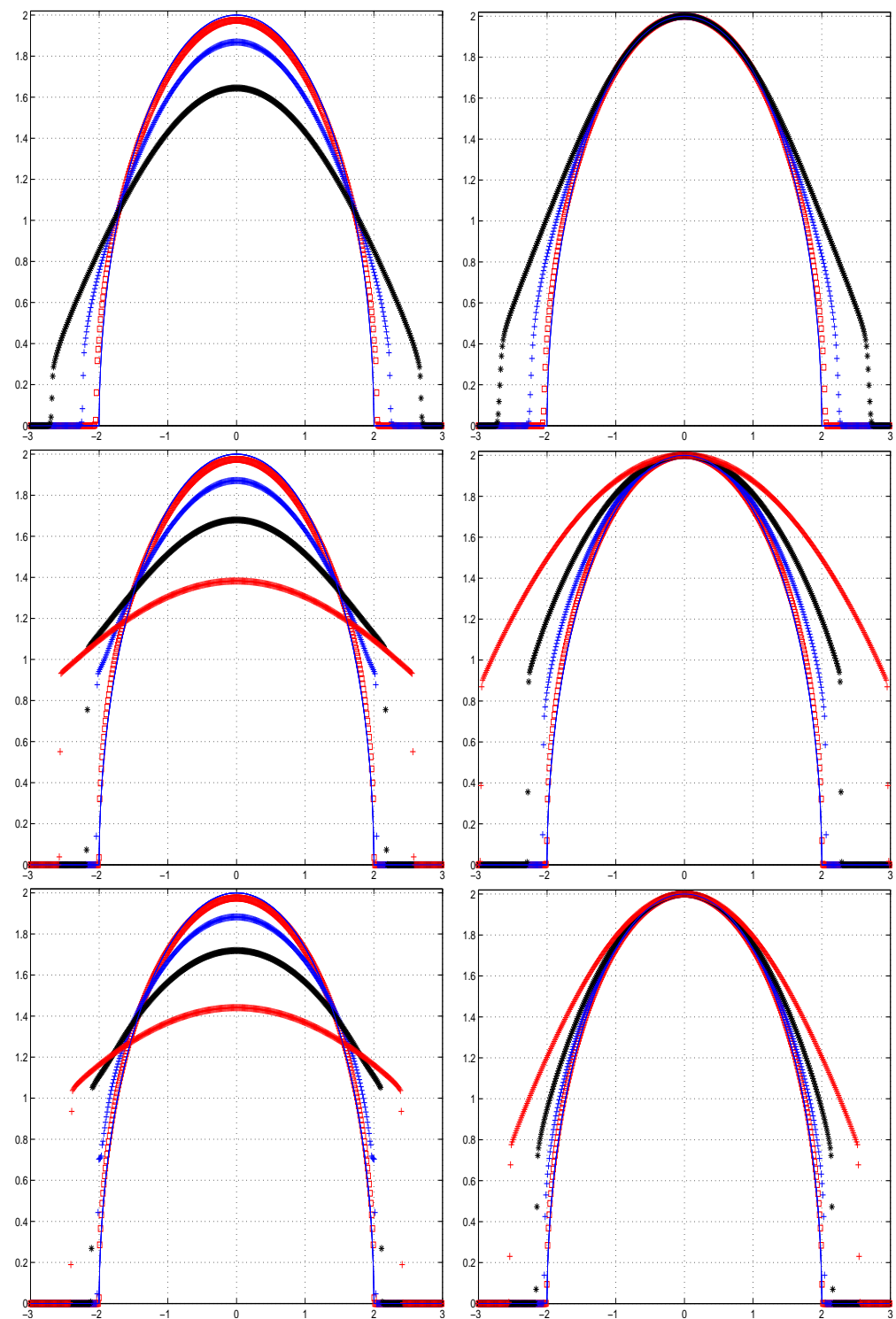

Figure 2: Semi-circle initial data. Evolution at times $t=0.05,0.25,0.7$ and $t=2$ represented with symbols red "square", blue "+", black "*" and red "+". Top to bottom, relativistic heat, porous media and limited speed porous media equations. Left column, limited diffusion equations, right column, Hamilton-Jacobi counterparts. 

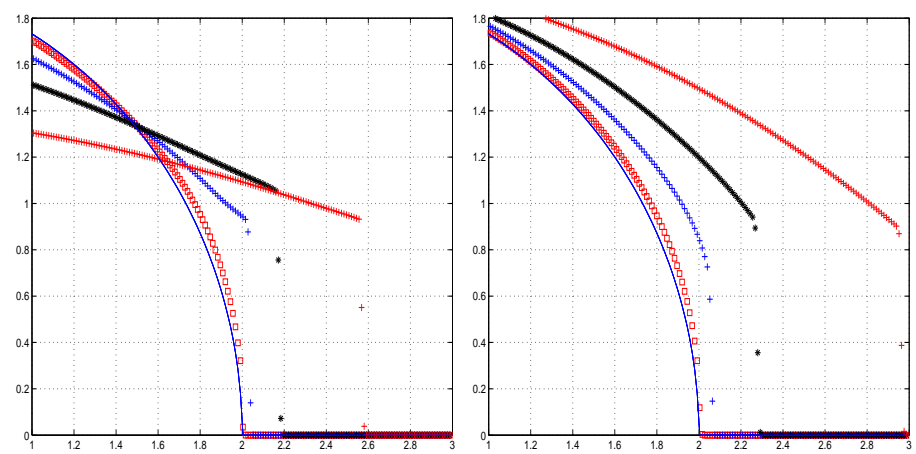

Figure 3: Semi-circle initial data. Zoomed region of second row of Figure 2. Porous media equation case at evolution at times $t=0.05,0.25,0.7$ and $t=2$ represented with symbols red "square", blue"+", black "*" and red "+". porous media Left, limited diffusion equations, right, Hamilton-Jacobi counterparts.

$$
u_{0}(x)= \begin{cases}\sqrt{4-x^{2}} & -2 \leq x \leq 2 \\ 0 & \text { elsewhere }\end{cases}
$$

This is a case of continuous function with discontinuities in first derivative such that the slopes at the boundaries of the support are vertical. This feature causes the creation of jump discontinuities at the boundaries of the support from the beginning of the evolution.

Figure 2 displays the approximate solutions at times $t=0.05,0.25,0.7$ and $t=2$ for the limited diffusion and Hamilton-Jacobi models and Figure 3 shows a zoomed region of second row of Figure 2.

We observe that the two porous media type models develop discontinuous diffusion fronts at the boundaries of the support from the beginning of the evolution. For the relativistic heat equation model, the associated hyperbolic flux is linear and the separation of the characteristic curves at the vertical angle generates a linear discontinuity traveling with constant speed. The cases of the porous media and limited speed porous media models join a nonlinear hyperbolic flux and the characteristic curves at the vertical angle intersect generating shock waves that propagate with signal dependent speed.

The corresponding Hamilton-Jacobi equations behave similarly at the boundaries although without any diffusion in the interior of the support contrarily to what occurs at the corresponding limited diffusion equations. 

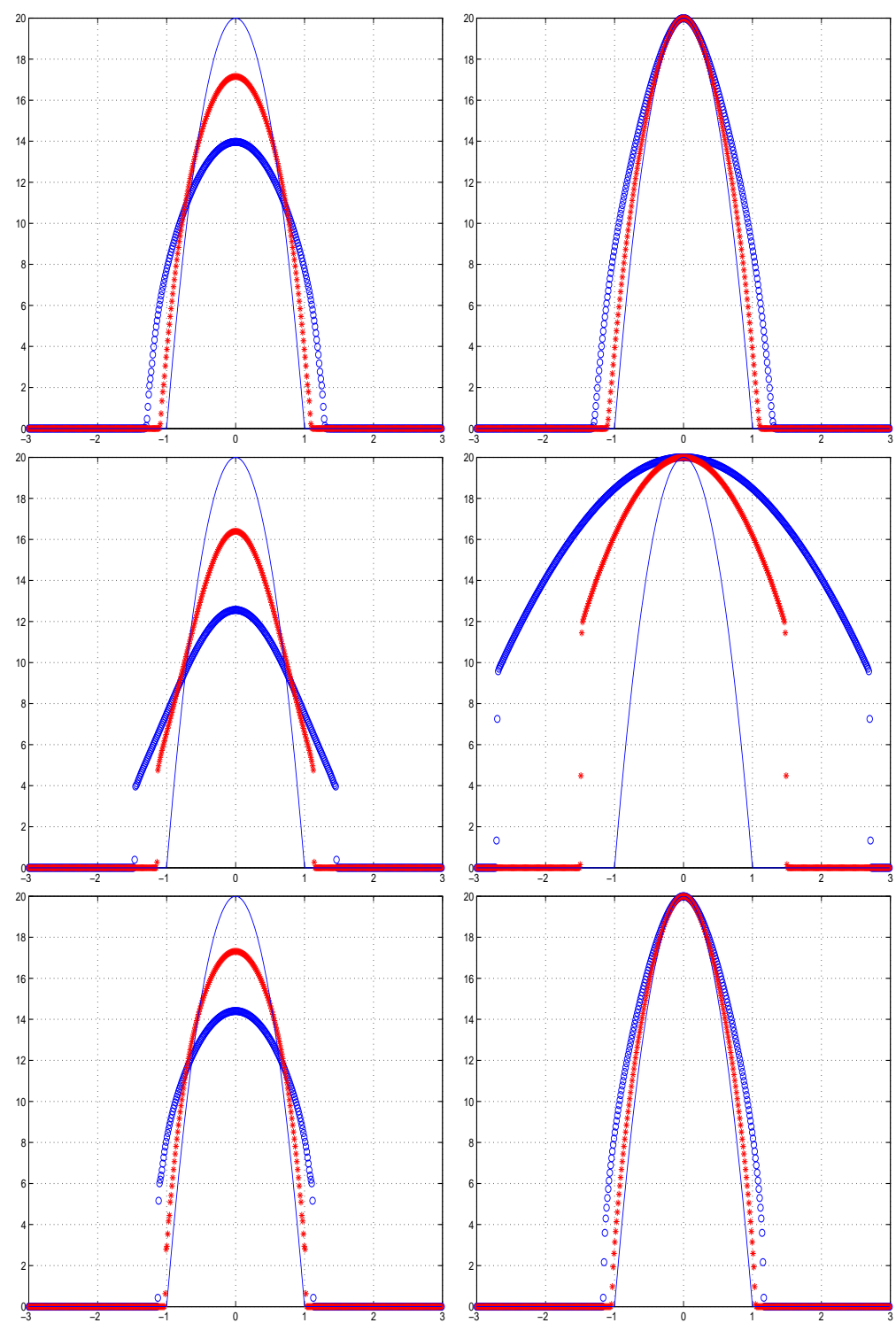

Figure 4: Continuous initial data. Evolution at times $t=0.1$, "*" and $t=0.3$ "o". Top to bottom, relativistic heat, porous media and limited speed porous media equations. Left column, limited diffusion equations, right column, Hamilton-Jacobi counterparts. 

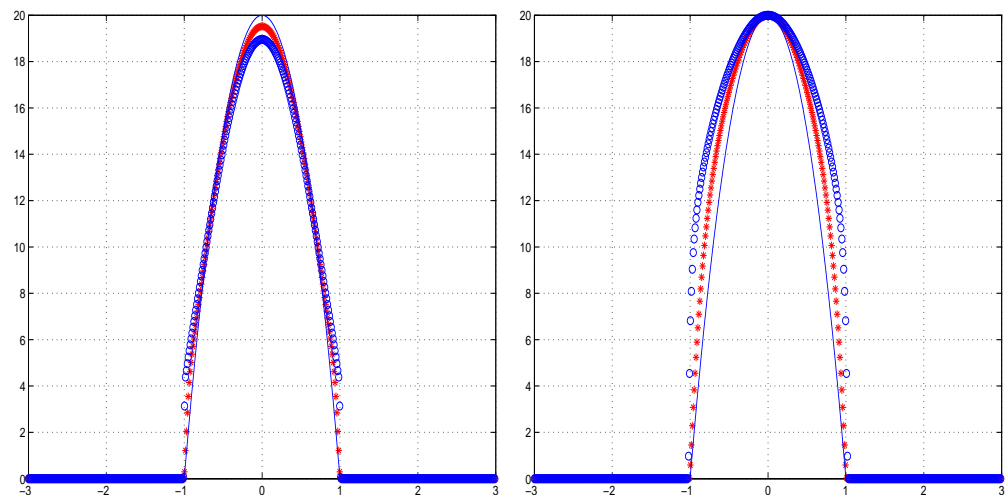

Figure 5: Continuous initial data. Evolution at times $t=0.012$, “*” and $t=0.022$ "o". porous media equation. Left, limited diffusion equations, right, Hamilton-Jacobi counterpart.

\subsection{Example 3: Continuous initial data}

We consider a continuous initial data with compact support. The slope of the function at the boundary of the support is finite i.e., the contact angle is not vertical. The initial data is defined as

$$
u_{0}(x)= \begin{cases}0 & |x| \geq 1 \\ 20 \max \left(1-x^{2}, 0\right) & |x|<1\end{cases}
$$

Numerical results are displayed in Figure 4. Similarly to the presentation of results of previous examples, left column includes the approximate solutions to the limited diffusion Fokker-Planck versions of each model problem and right column includes their Hamilton-Jacobi counterparts. From top to bottom the models are: relativistic heat, porous media and limited speed porous media equations. We plot in each picture the evolution at two times, $t=0.1$ and $t=0.3$ represented in red with "*" symbol and blue with "o" symbol respectively.

We observe in the first row that relativistic heat equation cases expand the support with constant velocity $C=1$ keeping the solution continuous.

Second and third rows correspond to the cases of limited diffusion porous media and limited speed porous media equations for which the hyperbolic fluxes are non linear. The dynamics of the evolution are similar and occur in two stages. First stage lasts a period of time called "waiting time" where the support of the signal does not expand while contact angles grow at both sides until they reach the vertical position. From this moment the solutions 
break due to the fact that the characteristics intersect and shock waves are generated at both sides of the boundary. This behavior is the same as the one of the evolution of the initial data in Example 2 where the initial contact angle is vertical.

The waiting time period for the porous media limited diffusion model (second row of Figure 4 ) is measured as $t \approx 0.015$. Figure 5 shows early times of this evolution. One at time $t=0.012$ just previous to reach vertical angle at the boundaries of the support and another where the shock has already developed, $t=0.022$.

While the evolution for both limited diffusion porous media share a similar dynamics, the speed of propagation of diffusion fronts are quite different in both cases. In the left picture of second row in Figure 4 we observe that propagation fronts for the porous media Fokker-Planck model travel at both sides a distance equal to 0.47 in a period of time that lasts $t \approx$ $0.3-0.015=0.285$ which is the total time minus the waiting time to break in shocks. Therefore average speed of the propagating front in those runs is $0.470 / 0.285=1.649$ which exceeds the established reference speed $C=1$. In the third row in Figure 4, the case of the limited speed porous media limited diffusion equation, we observe that the diffusion fronts expand the support with speed bounded by $C=1$.

For the case of the associated Hamilton-Jacobi equations (right column) the dynamics are similar. It can be observed that shock waves travel faster than in the corresponding limited diffusion equations due to the fact that jump discontinuities are bigger because of the lack of dissipation.

\section{Conclusions}

We have studied a class of limited diffusion Fokker-Planck equations and their associated Hamiltonians. We have defined a new class of HamiltonJacobi equations as the convective part of the Fokker-Planck equations and the cause of the formation of shocks in the solution of the limited diffusion equations. We propose a suitable finite difference numerical scheme to approximate the viscosity solution with shocks arising in the new class of Hamilton-Jacobi equations. The numerical scheme is designed in a way that combines the properties of classical numerical schemes for Hamilton-Jacobi equations together with the features of a shock capturing scheme to ensure correct propagation of waves. 
We analyze three model problems covering three different scenarios of the dynamics of front propagation. Two of the models are considered from the literature: one prescribing a constant speed of propagation of diffusion fronts and a second case where diffusion fronts propagate with unbounded finite density dependent speed. We propose a third model where diffusion fronts propagate with bounded density dependence speed. We perform a set of numerical experiments and compare the solution for the three model problems for the limited diffusion equations and their Hamilton-Jacobi counterparts. Asymptotic analysis and numerical examples show the inadequacy of standard porous media model to propagate fronts with limited speed. The results demonstrate the capability of the proposed limited speed porous media equation to bound the speed of propagation of fronts by an established reference speed.

\section{Acknowledgments}

First author acknowledges financial support by projects MICINN "Programa Ramón y Cajal", MICINN - MTM2011-28043, MTM2011-26995-C0201 and SGR2009-0345. Second author acknowledges support by MICINN MTM2011-28043.

\section{References}

[1] F. Andreu, V. Caselles, JM Mazon and S. Moll, Finite propagation speed for limited flux diffusion equation. Arch. Ration. Mech. Anal., 182, (2006), 269-297.

[2] F. Andreu, V. Caselles and JM Mazon, Some regularity results on the relativistic heat equation, J. Differential Eqn., 245, (2008), 3639-3663.

[3] Y. Brenier, Extended Monge-Kantorovich theory in Optimal transportation and applications: Lectures given at the CIME Summer School held in Martina Franca, Lectures Notes in Math. vol 1813, Springer-Verlag (2003), 91-122.

[4] V. Caselles, Convergence of the relativistic heat equation to the heat equation as $\mathrm{c} \rightarrow \infty$. Publ. Mat. 51 (2007), 121-142.

[5] V. Caselles, On the entropy conditions for some flux limited diffusion equations, J Diff. Eq, 250, 8, 3311-3348, 2011. 
[6] C. Cattaneo, Sulla conduzione del calore, Atti. del Semin. Matem., Unive di Modena, 3, pp 83-101 (1948-1949)

[7] A. Chertock, A. Kurganov, P. Rosenau, Formation of discontinuities in flux-saturated degenerate parabolic equations, Nonlinearity, 16, 18751898 (2003).

[8] R. Courant, K. Friedrichs, H. Lewy, Partial differential equations of mathematical physics, Math. Ann., 100, 32-74, 1928.

[9] M.G. Crandall, H. Ishii, P.L. Lions, Users guide to viscosity solutions of 2-nd order partial differential equations, Bull. Amer. Math. Soc., 27, 1, $1-67,1992$.

[10] J. Fourier, Theorie analytique de la chaleur (1822)

[11] S.K. Godunov, Difference methods for the numerical calculation of the equations of fluid dynamics, Math. Sbornik 47 (1959) 271306.

[12] A.C. Gurtin and M.E. Pipkin, A general theory of heat conduction with finite wave speeds, Arch. Rat. Mech. and Anal., 31, 113-126 (1968)

[13] D. Joseph, L. Preziosi, Heat waves, Rev. Mod. Phys., 41-73 (1989)

[14] L. D. Landau and E. M. Lifshitz. Fluid Mechanics, Second Edition: Volume 6 (Course in Theoretical Physics), Pergamon Press, Oxford, (1987).

[15] P.D. Lax, Hyperbolic systems of conservation laws and the mathematical theory of shocks waves SIAM, Philadelphia, 1973.

[16] R.J. LeVeque, Numerical Methods for Conservation Laws, Birkhauser Verlag, Zuerich, 1990.

[17] H. A. Levine, MP McGee S. Serna, Diffusion and reaction in the cell glycocalyx and the extracellular matrix, J. Math. Biology, 60, 1, 1-26, 2010 .

[18] A. Marquina, Diffusion front capturing schemes for a class of FokkerPlanck equations: application to the relativistic heat equation, J. Comput. Phys., 229, (2010) 2659-2674 
[19] D. Mihalas, B. Mihalas, Foundations of Radiation Hydrodynamics, Oxford University Press, Oxford (1984).

[20] S. J. Osher and J. Sethian , Fronts propagating with curvature dependent speed: Algorithms based on Hamilton-Jacobi formulations. J. Comput. Phys. 79, 12-49, 1988.

[21] S. J. Osher and C-W. Shu, High-order essentially non-oscillatory schemes for Hamilton-Jacobi equations, SIAM J. Numer. Anal., 28, (1991), pp. 907-922.

[22] P. Rosenau, Tempered diffusion: A transport process with propagation front and inertial delay, Phys. Rev. A 46 (1992) 7371-7374

[23] L. Rudin, S. Osher, E. Fatemi, Nonlinear total variation base noise removal algorithms, Physica D 60 (14) (1992) 259268.

[24] S. Serna, A. Marquina, Power ENO methods: a fifth-order accurate Weighted Power ENO method, J. Comput. Phys., 194, 2, 632-658, 2004.

[25] S. Serna and J. Qian. Fifth order Weighted Power-ENO schemes for Hamilton-Jacobi equations. J. Sci. Comp., 29:5781, 2006.

[26] C.W. Shu, S Osher, Efficient implementation of essentially nonoscillatory shock-capturing schemes, 2, J. Comput. Phys., 83, 1,(1989) 32-78.

[27] P.E. Souganidis, Approximation schemes for viscosity solutions of Hamilton- Jacobi equations. J. Diff. Eqn. 59, 143, (1985). 